\title{
2-Furyloxiranes III. Chain Extension with Different Polyols
}

\author{
Sami Boufi, Mohamed Naceur BelgaceM, and Alessandro GANDINI ${ }^{\dagger}$ \\ Matériaux Polymères, Ecole Française de Papeterie et des Industries \\ Graphiques (INPG), BP 65, 38402, Saint Martin d'Hères, France
}

(Received September 9, 1996)

\begin{abstract}
Furyl-, 5-methyl-2-furyl-, and $\alpha$-methyl-2-furyl oxiranes were used for the chain extension of different polyols and the reaction mechanism leading to the formation of the extented products was established. In all cases, it was shown that the chain extension was accompanied by a homopolymerization of the furanic oxiranes giving oligomers with degrees of polymerization of 6-7. The importance of transfer reactions during the propagation, namely the elimination of water and the subsequent anomalous formation of $\mathrm{OH}$ groups, was investigated in detail. This included the use of ${ }^{13} \mathrm{C} \mathrm{NMR}$ spectroscopy which confirmed the proposed mechanism and also showed the presence of $\mathrm{C}=\mathrm{C}$ unsaturations.

KEY WORDS Ring-Opening Polymerization / 2-Furyloxirane / OH-Promoted Initiation / Chain-

Extension / Transfer Reactions /
\end{abstract}

Within the framework of our long-standing interest in developing new macromolecular materials from renewable resources, ${ }^{1}$ and more specifically the vegetal biomass, as an alternative to fossil sources, furanic monomers, obtained as second-generation products from sugars, oligosaccharides, and polysaccharides via furfural and hydroxymethylfurfural, can give polymeric materials possessing interesting physical and chemical properties. $^{1-3}$

Among the numerous structures investigated, we devoted much attention to the synthesis and characterization of a variety of furanic polyurethanes ${ }^{4,5}$ and showed that they possess promising thermal and mechanical properties. The furan heterocycle was introduced through diols, diisocyanates or both types of monomers and acquired different configurations, namely within the chain backbones and/or pendant to them.

To the best of our knowledge, the polymerization of 2-furyloxirane (FO) was only studied in our laboratory and anionic systems proved to be the most adequate ${ }^{6,7}$ for the preparation of a regular polymer in high yields. A peculiarity of this monomer was that it could be activated simply by the presence of $\mathrm{OH}$ moieties, including traces of water, without the need of a more nucleophilic catalyst. ${ }^{8}$ This unique reactivity of the oxirane function, not shared by aliphatic or aromatic counterparts, is undoubtedly due to the electronic effect brought about by the neighbouring furan heterocycle with its dienic character, because neither aliphatic nor aromatic oxiranes display such a behavior. An obvious implication of this feature was the possibility of using FO to prepare block copolymers from macrodiols and graft copolymers from $\mathrm{OH}$-bearing polymers like poly(vinyl alcohol) or cellulosics. ${ }^{8}$ These reactions were indeed carried out successfully, but the products also contained some FO oligomers. Another way of making use of this unique reactivity was to synthesize new furanic polyols by simply mixing $\mathrm{FO}$ with various diols and triols for the elaboration of novel furanic polyurethanes. ${ }^{8}$

This paper constitutes the third part of an investigation $^{7,8}$ dealing with the specific chemical characteristics of FO. It is devoted to the study of the reaction mechanism of the latter (and of two homologous furanic oxiranes) with various $\mathrm{OH}$-bearing compounds in the absence of nucleophilic catalysts with the aim of understanding why the actual polymerization induced by the hydroxy groups is accompanied by the formation of large amounts of the oxirane homopolymer.

\section{EXPERIMENTAL}

The following abbreviations will be used in this work: FO , 2-furyloxirane

MFO , 5-methyl 2-furyloxirane

FMO , $\alpha$-methyl 2-furyloxirane

THMP, trihydroxymethyl propane

EG , ethylene glycol

BHMF, 2,5-bis(hydroxymethyl)furan

POET, poly(oxiethylene triol)s

NP , neopenthyl alcohol

PBT , "polybutyral", i.e., poly(vinyl alcohol)acetalyzed at $90 \%$ with butyral, $M_{w}=33000$

PFO, poly(2-furyloxirane)

\section{Materials}

A large sample of FO was generously provided by our colleagues at the Laboratoire de Chimie des Agroressources, ${ }^{9}$ of Toulouse National Polytechnic Institute to whom we are thankful. FO was purified and characterized in detail in our laboratory as previously reported. ${ }^{7}$ MFO and FMO were synthesized following the procedure described by Borredon et al., ${ }^{9}$ which consisted in reacting trimethylsulfonium iodide with 2-acetyl furan and 5-methyl furfural, respectively, in acetonitrile in the presence of potassium hydroxide at $50^{\circ} \mathrm{C}$. These oxiranes were purified by fractional distillation, dried over calcium hydride and characterized by FT-IR and NMR spectroscopy and mass spectrometry. All these spectra were in agreement with the expected structures. The ${ }^{1} \mathrm{H}$ NMR peaks of $\mathrm{MFO}$ and FMO are given with the following format: (assignment $=\delta(\mathrm{ppm})$, multiplicity, intensity, coupling constant $J(\mathrm{~Hz}))$ 
<smiles>[R14]Oc1ccc(C)o1</smiles>

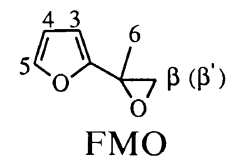

For $\mathrm{MFO}, \mathrm{H}^{3}=6.31, \mathrm{~d}, 1, J_{3-4}=3.1 ; \mathrm{H}^{4}=5.92, \mathrm{~m}, 1$, $J_{4-3}=4.0 ; \mathrm{H}^{\alpha}=3.80, \mathrm{q}, 1, J_{\alpha-\beta\left(\beta^{\prime}\right)}=7.0 ; \mathrm{H}^{\beta-\beta^{\prime}}=3.10$ and 3.24 , q, q, $2, J_{\beta^{\prime}-\beta}=8.1, J_{\beta-\beta^{\prime}}=9.5 ; \mathrm{H}^{5}=2.25, \mathrm{~d}, 3$, $J_{5-3}=0.7$.

For MFO, $\mathrm{H}^{5}=7.31, \mathrm{t}, 1, J_{5-3}=1.8 ; \mathrm{H}^{3-4}=6.30-6.50$, A-B system, $2 ; \mathrm{H}^{\beta-\beta^{\prime}}=2.92$ and $3.32, \mathrm{~d}, \mathrm{~d}, 2, J_{\beta^{\prime}-\beta}=5.4$, $J_{\beta-\beta^{\prime}}=5.3 ; \mathrm{H}^{6}=1.67, \mathrm{~s}, 3$.

The elemental analyses of these two monomers were also carried out. The calculated values for both oxiranes are $67.7,6.5$, and $25.8 \%$ for $\mathrm{C}, \mathrm{H}$, and $\mathrm{O}$, respectively. The corresponding experimental data were in very good agreement, namely: (i) for MFO, 67.9, 6.5, and 25.9\%; (ii) for FMO, 68.1, 6.4, and 25.6\%.

Finally, the mass spectra of these two monomers gave the expected $\mathrm{M}^{+}$at 124 and the expected fragments corresponding to (i) the cleavage between the two heterocycles, (ii) the loss of the methyl substituent, and (iii) the typical furanic pattern giving $\mathrm{CO}$ and various $\mathrm{C}_{3}$ derivatives.

Several polyols with different functionalities were used for chain extension with the furanic oxiranes. The characteristics of these reagents are resumed in Table I.

All other reagents, catalysts and solvents used in this investigation were commercial dry products of the highest purity available.

\section{Reaction Conditions}

The chain extensions of the oxiranes were carried out under nitrogen by adding the given alcohol or polyol to an excess of the chosen furanic oxirane in different proportions in bulk, in different solvents and at $40^{\circ} \mathrm{C}$, except when stated otherwise.

Typically, $1 \mathrm{~g}$ of the hydroxylic reagent and the excess quantity of the given oxirane were mixed, with or without solvent, under magnetic stirring in a flask kept under a nitrogen atmosphere and immersed in a constant temperature bath. At the end of the reaction, the ensuing products were recovered by precipitation in methanol or vacuum removal of the unreacted oxirane (and solvent), depending on their molecular weight. The specific quantitative conditions of these reactions are given in the corresponding Tables below.

\section{Characterization}

The products obtained in the different reactions described above were characterized by ${ }^{1} \mathrm{H}$ and ${ }^{13} \mathrm{C}$ NMR using a Brucker 300 apparatus working at 300 and 75 $\mathrm{MHz}$, respectively and by FT-IR spectroscopy with a Perkin-Elmer Paragon 1000 spectrometer. Their molecular weights were determined by high-performance liquid chromatography (HPLC), size exclusion chromatography (SEC) and vapor-pressure osmometry (Knauer). The chromatograms were recorded on a Knauer GPC apparatus equipped with two columns (100 and $1000 \AA$ ) connected in series and two detectors: a UV sensor working at $254 \mathrm{~nm}$ and a refractometer. The calibration curve was obtained using standard polystyrene samples. Mass spectrometry (Nermag R 10 10C) working in a
Table I. Chemical structure and average functionality of the polyols used for the chain-extention experiments with FO

Precursors

${ }^{a} F$, average functionality.

FAB mode was also used for determining molecular weights of oligomers. Mass spectrometry working in the ionisation mode and coupled with a GLC chromatograph (Delsi DI 700) was used to determine the molar masses and fragmentation patterns of MFO and FMO.

The hydroxy index or number $\left(I_{\mathrm{OH}}\right)$ is an important parameter in the characterization of the hydroxy content of polyols. By definition this parameter is the number of milligrams of potassium hydroxide equivalent to the hydroxy content of $1 \mathrm{~g}$ of the polyol. $I_{\mathrm{OH}}$ was determined following the ASTM D1638 standard, which consists in dissolving the product in pyridine, treating it with a known excess of phthalic anhydride under reflux for one hour and back-titrating the unreacted mixture of acid and anhydride. The titration of these solutions were carried out using an automatic potentiometric titrator (Mettler DL 21) equipped with a glass DG 111 electrode.

\section{RESULTS AND DISCUSSION}

\section{Molecular Weights and Transfer Reactions}

In order to examine the mechanisms leading to chain extension and homopolymer, different precursors were made to react with FO, as shown in Table I. Theoretical molecular weights of the chain-extended products were calculated from eq 1, assuming that the polymerization started from the $\mathrm{OH}$ groups and that no transfer reaction occurred:

$$
M_{n}=M_{p}+n \times 110
$$

where $M_{n}$ is theoretical number-average molecular weight of the chain-extended product, $n$ the molar ratio $\mathrm{FO} /$ Precursor, and $M_{p}$ the molecular weight of the precursor used, viz., the diol or polyol.

The differences between the calculated and determined values of $M_{n}$ (Table II) confirmed the relevance of the transfer reactions. Indeed, the relative amount of FO 
Table II. Characteristics of polyols chain-extended by FO

\begin{tabular}{|c|c|c|c|c|c|c|}
\hline \multirow{2}{*}{ Precursors } & \multirow{2}{*}{$\frac{\mathrm{FO} / \text { Precursor }}{\text { molar ratio }}$} & \multirow{2}{*}{$\frac{\text { Reaction time }}{\text { days }}$} & \multirow{2}{*}{$\frac{\text { Yields }}{\%}$} & \multirow{2}{*}{$M_{n}(\text { th })^{\mathrm{a}}$} & \multirow{2}{*}{$M_{n}(\operatorname{det})^{\mathbf{b}}$} & \multirow{2}{*}{$F^{\mathrm{c}}$} \\
\hline & & & & & & \\
\hline \multirow[t]{6}{*}{ THMP } & 3 & 1 & 100 & 460 & 405 & 2.95 \\
\hline & 6 & 2 & 100 & 790 & 500 & 2.70 \\
\hline & 9 & 2 & 100 & 1120 & 610 & 2.65 \\
\hline & 15 & 2 & 100 & 1780 & 670 & 2.60 \\
\hline & 30 & 3 & 100 & 3500 & 680 & 2.40 \\
\hline & 45 & 3 & 100 & 5080 & 650 & 2.15 \\
\hline THMP $^{\mathrm{d}}$ & 22 & 7 & 100 & 2544 & 675 & - \\
\hline EG & 12 & 3 & 100 & 1382 & 590 & 2.05 \\
\hline \multirow[t]{2}{*}{ BHMF } & 4 & 1 & 100 & 568 & 440 & 2.08 \\
\hline & 30 & 3 & 100 & 3428 & 700 & 2.04 \\
\hline \multirow[t]{2}{*}{ POET } & 6 & 5 & 95 & 3150 & 1800 & 2.80 \\
\hline & 12 & 10 & 90 & 3820 & 1400 & 2.50 \\
\hline FO & \multicolumn{6}{|c|}{ No changes after 20 days } \\
\hline
\end{tabular}

${ }^{a}$ Calculated from eq $1 .{ }^{b}$ Determined by VPO. ${ }^{\mathrm{c}}$ Average OH-functionality calculated from eq $2 .{ }^{\mathrm{d}}$ Reaction was carried out under vacuum of $10^{-5} \mathrm{~mm} \mathrm{Hg}$.

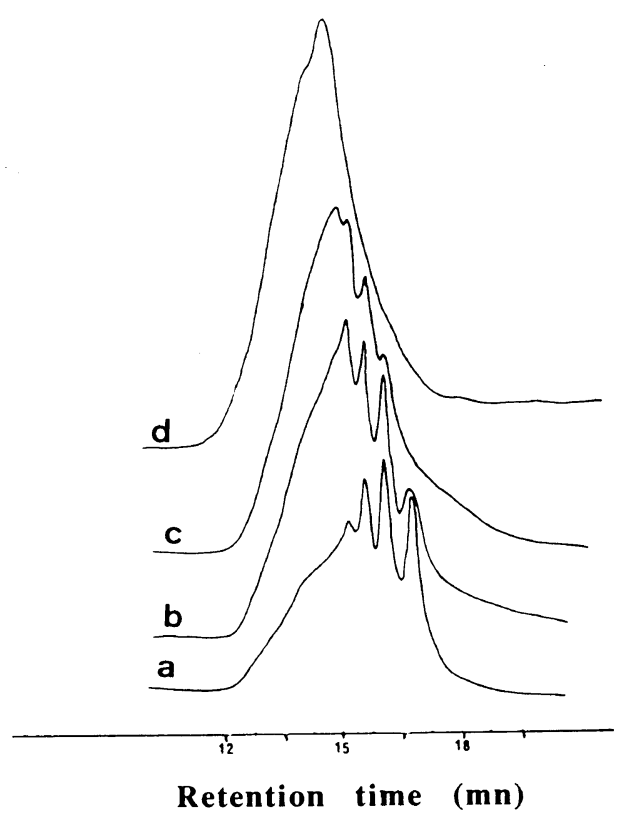

Figure 1. SEC of FO+THMP: $a, 3 / 1 ; b, 6 / 1 ; c, 9 / 1 ; d, 30 / 1$.

homopolymer must have been high, since a molecular weight around 600 was systematically obtained, independent of both the precursor structure and the molar ratio $n$.

FO-extented THMP prepared with different [FO]/ [THMP] ratios were also characterized by SEC, as shown in Figure 1. As the relative amount of FO was increased, the molecular weight of the product tended to increase (see also Table II), but mostly the contribution of individual oligomers decreased to be replaced progressively by a single broader peak. Similar features were observed with diols, as shown in Table I. The decrease in $M_{n}$ when POET was treated with FO (see Table II) indicates that chain extension must have been accompanied by a large contribution from transfer reactions. The chain extention of PBT indicated again the predominance of transfer in these systems. The SEC of this grafted material is given in Figure 2 and shows that the most important product formed was eluted with the same retention time as those formed with smaller

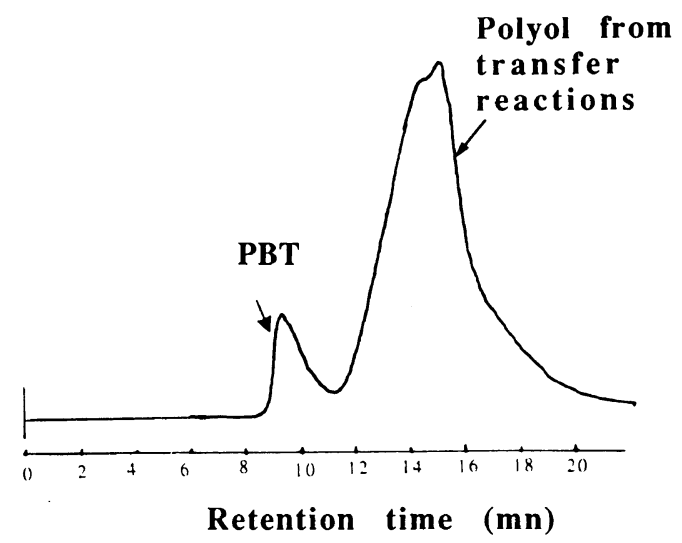

Figure 2. $\mathrm{SEC}$ of $\mathrm{FO}+\mathrm{PBT}(3 / 1)$.

chain-extended diols and triols.

Under the conditions chosen here, FO did not homopolymerize in the absence of an $\mathrm{OH}$-bearing additive, as shown in Table II. Moreover, we have previously given all the mechanistic evidence related to the possibility of initiating the FO polymerization from alcohols in the absence of any added or spurious catalyst. ${ }^{7,8}$ These facts, coupled with all the above evidence, points to a clear-cut kinetic situation: the uncatalyzed polymerization of FO is initiated by the hydroxy functions and the ensuing propagation occurs in the presence of a competitive transfer reaction in such a way that the ratio of the frequencies of the two events is about 6 , as suggested by the limiting molecular weight of products arising from small polyols.

\section{FAB Mass Spectrometry}

Figure 3 shows two FAB mass spectra corresponding to the same system with different initial molar ratios. The fact that the fragmentation pattern as well as the highest molecular peaks were essentially the same for both products, confirms that beyond the initial stages of these chain-extension reactions, the predominance of chain transfer gives rise to PFO oligomers bearing the same structure. Of course the proportion of chainextended material and oligomeric PFO changes as a function of the initial stoichiometric conditions, but the 

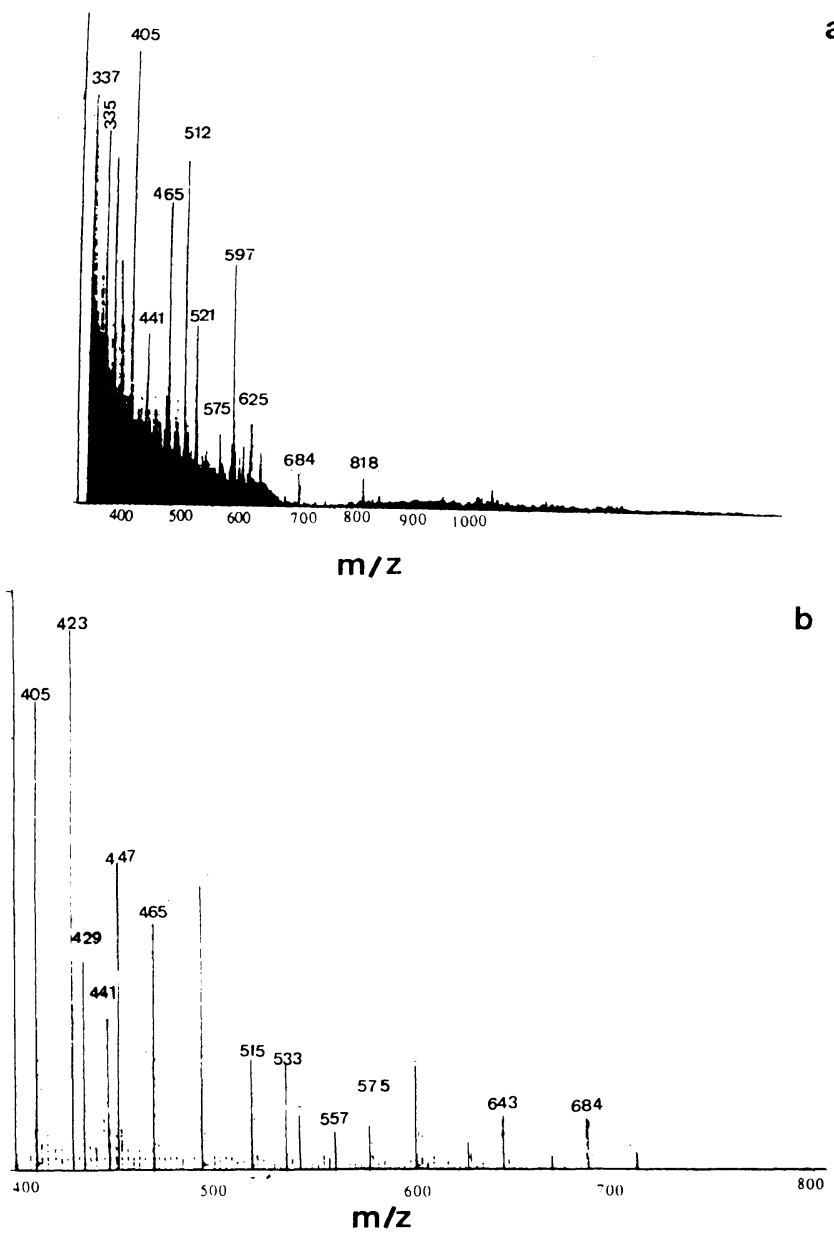

Figure 3. Mass spectra of (a) FO+THMP (6/1), (b) FO + THMP $(30 / 1)$.

Table III. Mass fragments of THMP chain-extended by FO and PFO homopolymers

\begin{tabular}{clll}
\hline $\begin{array}{c}\text { Fragment of } \\
\text { chain-extended } \\
\text { product }\end{array}$ & $\begin{array}{c}\text { Structure } \\
(\mathrm{FO} / \mathrm{THMP})^{\mathrm{a}}\end{array}$ & $\begin{array}{c}\text { Fragment of } \\
\text { homopolymer }\end{array}$ & $\begin{array}{c}\text { Structure } \\
(\mathrm{FO})_{n}\end{array}$ \\
\hline 575 & $4 / 1$ & 643 & $(\mathrm{FO})_{6}-\mathrm{H}_{2} \mathrm{O}$ \\
667 & $5 / 1-\mathrm{H}_{2} \mathrm{O}$ & 661 & $(\mathrm{FO})_{6}$ \\
684 & $5 / 1$ & 771 & $(\mathrm{FO})_{7}$ \\
337 & $2 / 1-\mathrm{H}_{2} \mathrm{O}$ & 331 & $(\mathrm{FO})_{3}$ \\
355 & $2 / 1$ & 405 & $(\mathrm{FO})_{4}-2 \mathrm{H}_{2} \mathrm{O}$ \\
429 & $3 / 1-2 \mathrm{H}_{2} \mathrm{O}$ & 423 & $(\mathrm{FO})_{4}-\mathrm{H}_{2} \mathrm{O}$ \\
447 & $3 / 1-\mathrm{H}_{2} \mathrm{O}$ & 441 & $(\mathrm{FO})_{4}$ \\
465 & $3 / 1$ & 515 & $(\mathrm{FO})_{5}-2 \mathrm{H}_{2} \mathrm{O}$ \\
521 & $4 / 1-3 \mathrm{H}_{2} \mathrm{O}$ & 533 & $(\mathrm{FO})_{5}-\mathrm{H}_{2} \mathrm{O}$ \\
539 & $4 / 1-2 \mathrm{H}_{2} \mathrm{O}$ & 551 & $(\mathrm{FO})_{5}$ \\
557 & $4 / 1-\mathrm{H}_{2} \mathrm{O}$ & 625 & $(\mathrm{FO})_{6}-2 \mathrm{H}_{2} \mathrm{O}$ \\
\hline
\end{tabular}

a THMP chain-extended by $\mathrm{FO}$ with $\mathrm{FO} / \mathrm{THMP}=\times / 1$.

nature of both products is not sensitive to that parameter. Table III gives details about the mass of the fragments and points to the existence of molecular entities corresponding to PFO deprived of one, two or three molecules of water. Moreover, the molecular weights were close to 700 , in excellent agreement with the values from VPO, reported in Table II.

\section{OH Functionality}

The average hydroxy functionality of the chain-extent-

a

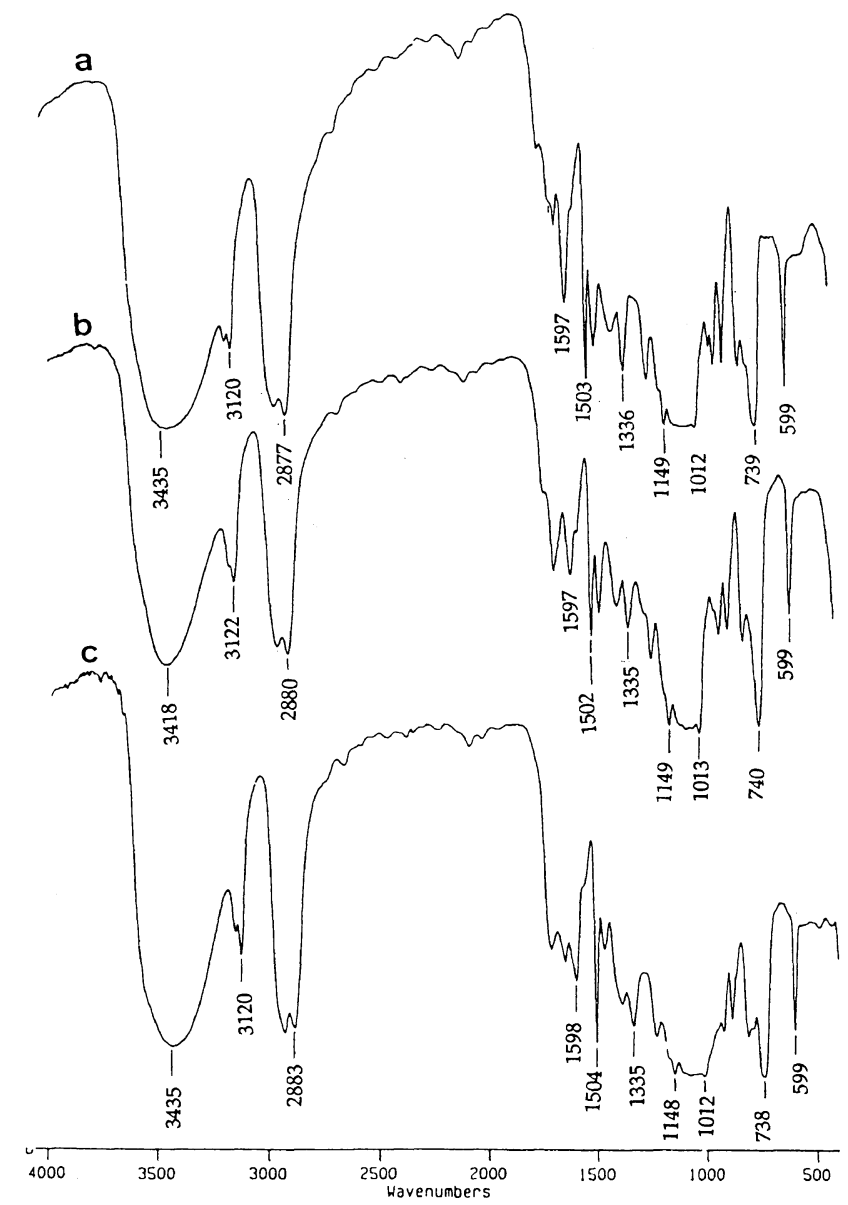

Figure 4. IR spectra of products from $\mathrm{FO}+\mathrm{THMP}$. [FO]/[THMP]: a, $3 / 1 ; b, 9 / 1 ; c, 30 / 1$.

ed polyols was determined using eq 2 :

$$
F_{n}=\frac{I_{\mathrm{OH}} \cdot M_{n}}{56100}
$$

where, $I_{\mathrm{OH}}$ is the hydroxy index and $M_{n}$ the numberaverage molecular weight of each product, as given in Table II. The average functionality of THMP extended by FO decreased from about 3 to values somewhat higher than 2 with increasing [FO]/[THMP] ratio, as shown in Table II. This trend was also observed in the case of the extention of POET by FO and is certainly due to the homopolymerization of FO, induced by transfer reactions. For the systems involving diols, $F_{n}$ remained close to 2 .

\section{FT-IR and ${ }^{1} H$ NMR Characterization}

Figure 4 shows three FT-IR spectra of products obtained with different $[\mathrm{FO}] /[\mathrm{THMP}]$ ratios, which show very similar features. In order to establish the nature of the transfer reaction(s), the FT-IR spectrum of a PFO prepared by a catalyzed anionic polymerization ${ }^{7}$ was compared with that of a sample of FO-extented THMP prepared with $[\mathrm{FO}] /[\mathrm{THMP}]=20$. As shown in Figure 5 , two spectral regions were compared, namely the $\mathrm{OH}+$ $\mathrm{CH}$ vibrational domain and that related to the $\mathrm{C}=\mathrm{O}+$ $\mathrm{C}-\mathrm{O}-\mathrm{C}+\mathrm{C}=\mathrm{C}$ groups. The most important differences were: (i) the intensity of the $\mathrm{OH}$ band at $3435 \mathrm{~cm}^{-1}$ which was vastly higher with the chain-extended product and (ii) three peaks situated at around 1650, 1600, and 

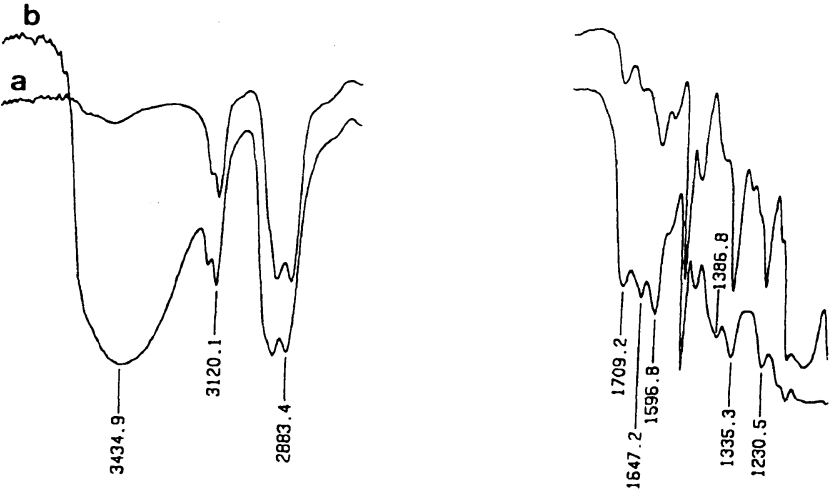

Figure 5. IR spectra of (a) PFO and (b) FO+THMP (3/1).
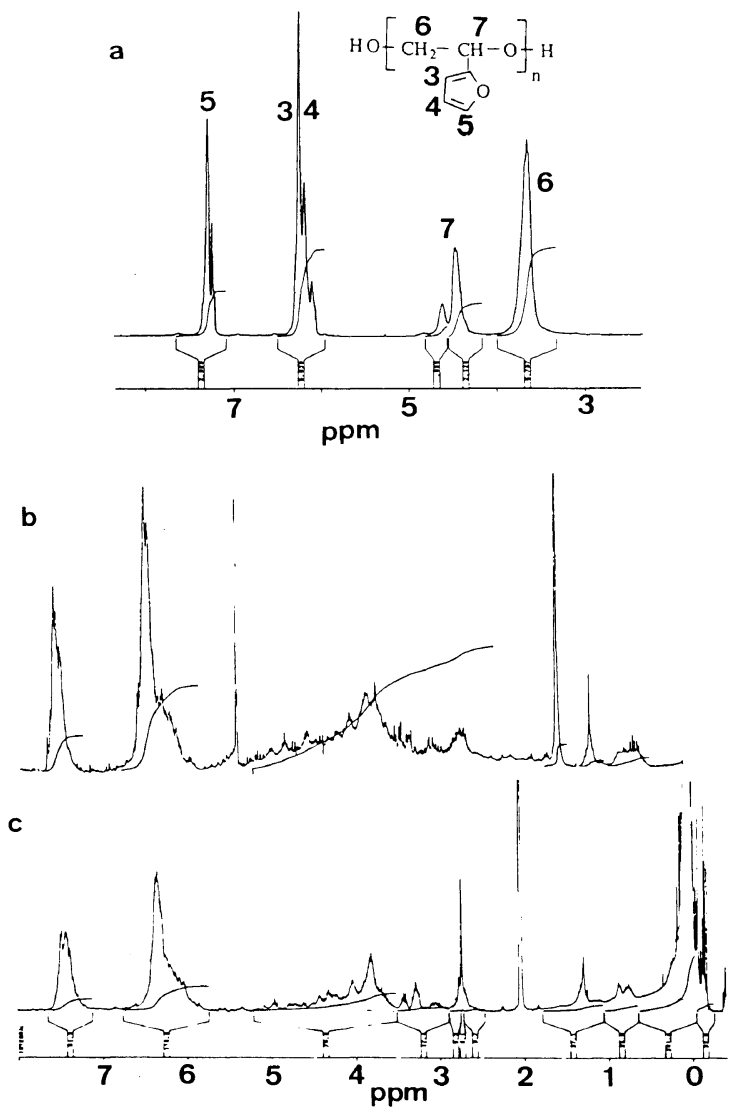

Figure 6. $\quad 300 \mathrm{MHz}{ }^{1} \mathrm{H}-\mathrm{NMR}$ spectra of (a) $\mathrm{PFO}$, (b) $\mathrm{FO}+\mathrm{THMP}$ (30/1), (c) FO+ THMP (20/1) after sylilation.

$1390 \mathrm{~cm}^{-1}$, respectively, followed the same trend, albeit with a less striking difference.

Figure 6 represents a comparison of the ${ }^{1} \mathrm{H}$ NMR spectra of: (i) a PFO with $M_{n}=1800$ prepared by a catalyzed anionic polymerization, ${ }^{7}$ (ii) a product from the system $[\mathrm{FO}] /[\mathrm{THMP}]=30$, and (iii) a silylated product from the system $[\mathrm{FO}] /[\mathrm{THMP}]=20$. This silylation, carried out with $\mathrm{N}, \mathrm{O}$-bis(trimethylsilyl)trifluoroacetamide), induced the disappearance of the $\mathrm{OH}$ groups and simplified the corresponding ${ }^{1} \mathrm{H}$ NMR spectrum. The efficiency of silylation was evaluated by IR analysis. Figure 7 gives the spectrum of the silylated product shown in Figure 6 and clearly proves the absence of $\mathrm{OH}$ moieties by the corresponding absence of a band around $3400 \mathrm{~cm}^{-1}$. The main difference of the three spectra of Figure 6 is related to the resonance of the $\mathrm{H}^{\alpha}$ and $\mathrm{H}^{\beta}$ protons of PFO which give peaks at 3.6 and $4.2 \mathrm{ppm}$,

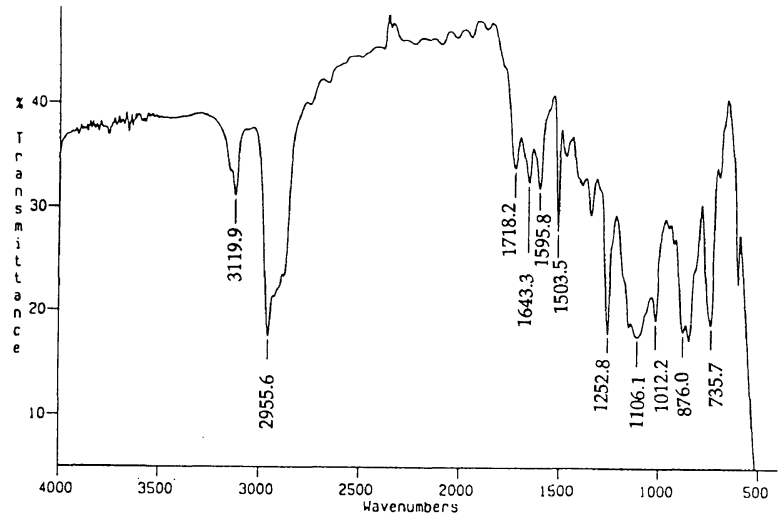

Figure 7. IR spectrum of sylilated FO+THMP (20/1).

respectively, with the "normal" polymer. ${ }^{7}$ In the same region, the products derived from THMP, but also from all the other polyols used in this work, gave a much less well-defined resonance pattern, as clearly visible in the corresponding spectra of Figure 6.

The quantitative comparison of spectrum $\mathbf{6 a}$ with $\mathbf{6 b}$ and $\mathbf{6 c}$ was particularly instructive. In fact, whereas for PFO the integrations reflect the expected regular polyether structure, ${ }^{7}$ major discrepancies appeared in the spectra of the products prepared here. Thus, at first sight, the relative intensity of $\mathrm{H} 5$ at $7.2 \mathrm{ppm}$, with respect to $\mathrm{H} 4+\mathrm{H} 3$ at $6.1 \mathrm{ppm}$, seemed to decrease, but the real trend was that a new resonance occurred just above $6 \mathrm{ppm}$ which artificially reinforced the $\mathrm{H} 3+\mathrm{H} 4$ peak. The strong asymmetric shape of the new peak suggests strongly that the right-hand shoulder is not related to the resonance of $\mathrm{H} 3$ or $\mathrm{H} 4$. It can be concluded therefore that the furanic protons are not involved in any side reaction during the synthesis of the chain-extended products, but that new structures appear which must contain alkenyl protons resonating around $6 \mathrm{ppm}$. Another important remark concerns the loss of $\mathrm{H}^{\alpha}$ and $\mathrm{H}^{\beta}$ protons, which could be quantified at 15 to $30 \%$, depending on the system, by the direct comparison between the spectra of the product as such and its silylated derivative, as shown in Figure 6.

The transfer reaction, observed in kinetic terms above, can now be examined under its mechanistic aspects, knowing that alkenyl structures appear to the detriment of aliphatic counterparts and that no such feature was ever observed in the catalysed anionic polymerization of FO. ${ }^{7}$ It is therefore necessary to postulate a reaction involving a polymer chain bearing a terminal $\mathrm{OH}$ group and a monomer, alternative to the molecular propaga$\operatorname{tion}^{8}$ (as opposed to the classical anionic propagation step of oxiranes ${ }^{7}$ ). This reaction pattern must not call upon external agents since we proved that runs conducted under atmospheric conditions or in vacuo with anhydrous reagents produced the same results (see Table II). The mechanisms proposed in Scheme 1 seem reasonable in this context and take into account the possible existence of two types of terminal $\mathrm{OH}$ arising from $\alpha$ and $\beta$-opening of the oxirane ring.

The experimental evidence imposed the formulation of a transfer mode in which a water molecule leaves the end group to add onto the monomer. Obviously, intermediate species must be involved in this mecha- 


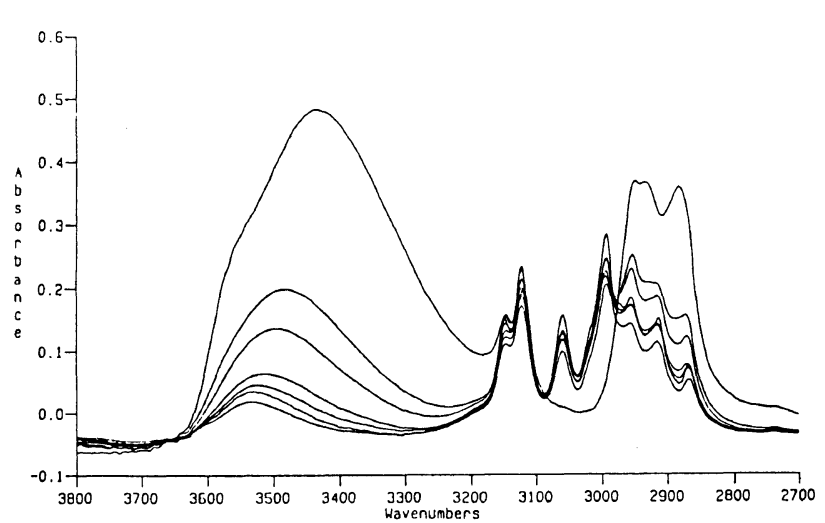

Figure 8. Kinetic evolution of the chain extension of NP by FO (1: 15 molar ratio) followed by FT-IR at $40^{\circ} \mathrm{C}$. See Figure 9 for the reaction times corresponding to each spectrum.

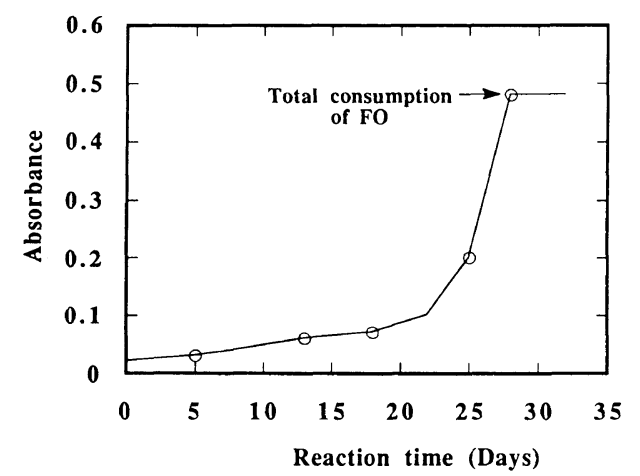

Figure 9. $\mathrm{OH}$ concentration vs. reaction time: data from Figure 8.

nisms, i.e., there is no actual release of a water molecule. What counts here is the fact that the overall interaction explains adequately, not only the very existence of transfer, but, more importantly, the increase in the $\mathrm{OH}$ content, as seen in the FT-IR spectra, and the progressive loss of aliphatic $\mathrm{H}^{\alpha}$ and $\mathrm{H}^{\beta}$ with the corresponding formation of unsaturated groups, as clearly detected by both FT-IR and ${ }^{1} \mathrm{H}$ NMR. It is worth noting that because the degrees of polymerization (DPs) are close to 6 in our systems, the relevance of these alkenyl end-groups is quite pronounced and easily detected by spectroscopic means. The mass spectrometry data constitute an additional piece of evidence in favor of the mechanisms proposed in Scheme 1 since they show the presence of oligomeric entities corresponding to PFO minus water molecules, as already pointed out and given in Table III. Of course, some of these fragments could have arisen from dehydration in the spectrometer, but the very fact that the loss of water molecules is so readily achieved is in itself an argument in support of the transfer we postulated.

In order to corroborate this mechanisms, we carried out an experiment in which NP was treated with a fifteenfold excess of FO in the usual conditions. Figure 8 shows the evolution of the FT-IR spectrum of the reaction mixture as a function of time. The most striking feature was of course the very pronounced relative increase of the $\mathrm{OH}$ band. This increase was particularly significant towards the end of the reaction, as indicated in Figure 9 which portrays the kinetics of $\mathrm{OH}$ formation. The exponential shape of this curve suggests an autocatalytic effect, viz., the higher the $\mathrm{OH}$ concentration the
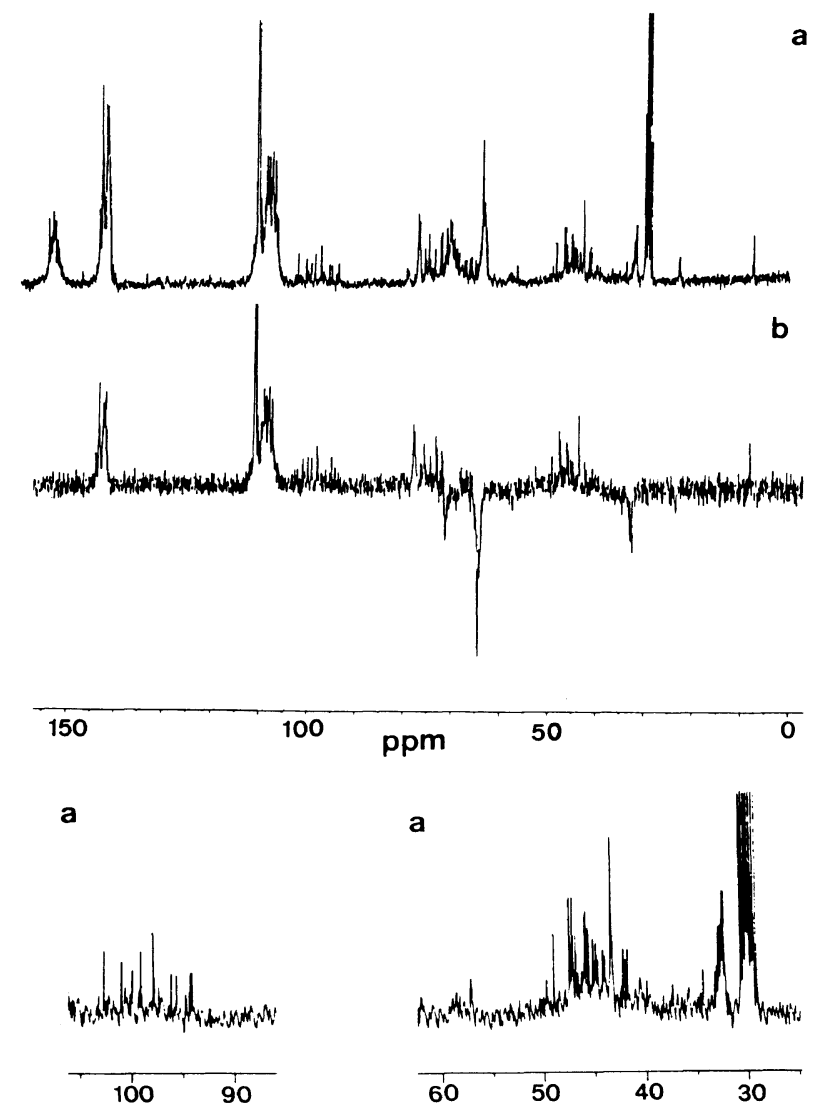

Figure 10. $75 \mathrm{MHz}{ }^{13} \mathrm{C}-\mathrm{NMR}$ spectra of FO+THMP (20/1): (a) as such and (b) in DEPT mode.

higher the rate of both propagation and transfer, the latter giving rise to further increase in the amount of hydroxy groups present. When the FO had been totally consumed, the increase in $\mathrm{OH}$ concentration ceased, indicating that this phenomenon requires the presence of monomer, as our mechanism postulates. The other features of the spectral evolution are the fact that the peaks attributed to the furan ring $\left(3100-3200 \mathrm{~cm}^{-1}\right)$ did not change appreciably, whereas the absorptions related to the oxirane moiety $\left(3000-3050 \mathrm{~cm}^{-1}\right)$ decreased progressively and finally vanished and were replaced by an increase in the peaks between 2850 and 2980 belonging to the aliphatic $\mathrm{CH}$ and $\mathrm{CH}_{2}$. All these observations are in tune with previously mentioned features and with the proposed mechanisms.

\section{${ }^{13}$ C NMR Spectra}

In order to find further evidence in favor of the suggested mechanism, we conducted some ${ }^{13} \mathrm{C}$ NMR analyses of representative products. Figure 10 shows one such spectrum in different modes and resolutions. The comparison between this spectrum and that of a PFO prepared by strong anionic initiators ${ }^{7}$ reveals two major differences, namely the presence of new peaks between 94 and 105 and in the $30-50 \mathrm{ppm}$ region. The former resonances can be attributed to unsaturated structures such as:
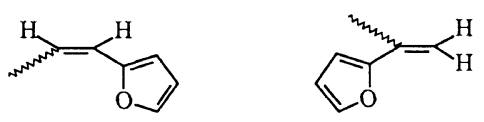


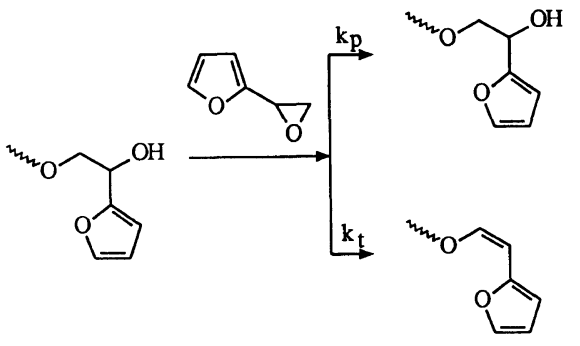

or

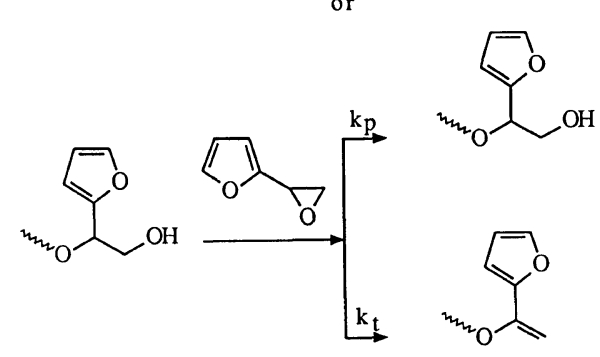

Scheme 1

since the corresponding DEPT spectrum shows no peak inversion. In addition, the specific resonance values suggest that these unsaturations are at the end of the oligomeric chains, i.e., each carbon atom bears a hydrogen atom, in accord with the transfer mechanism of Scheme 1. Peaks at $30-50 \mathrm{ppm}$ were identified by calculations ${ }^{10}$ of shifts using eq 3 :

$$
\delta_{\mathrm{c}}=-2.3-\sum z_{i}-\sum s_{i}+\sum k_{i}
$$

where $z_{i}$ is a constant which depends on the nature of the substituent, $s_{i}$ a steric hindrance correction factor and $k_{i}$ a conformation factor. These calculations demonstrated the presence of the following structures:
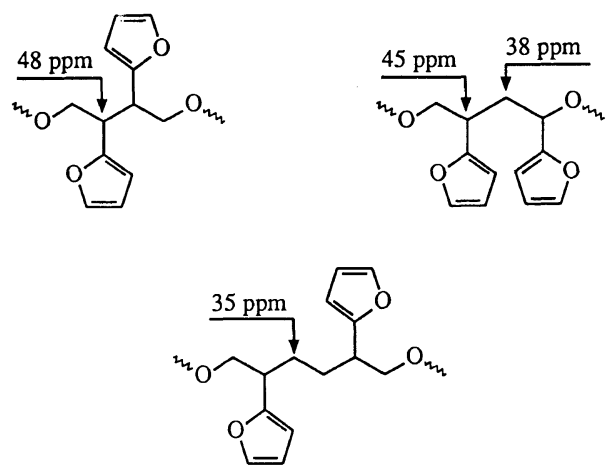

These results explain the presence of $\mathrm{CH}$ and $\mathrm{CH}_{2}$ moieties which are not linked to oxygen atoms, detected in the ${ }^{1} \mathrm{H}$ NMR spectra (Figure 6) in the form of peaks at $1.3-2.7 \mathrm{ppm}$. The origin of these structures is unclear, but could be attributed to the interaction between an oligomer bearing a terminal unsaturation (see the two relevant structures in Scheme 1) and the oxirane moiety of FO which can open either way.

\section{Other Furan Oxiranes}

The other furanic oxiranes, FMO and MFO, were also used as chain extender with THMP and EG. As shown in Table IV, except for the reaction time, which was reduced in both cases, the products obtained had lower molecular weights and $\mathrm{OH}$ functionality. The former values were confirmed by SEC and mass spectrometry. The IR and NMR spectra of these products showed features which were very similar to those obtained using FO as chain extender, except of course for the specific absorption of the methyl substituents. It can therefore be concluded that the use of these oxiranes did not change the qualitative pattern of behavior with respect to $\mathrm{FO}$. This rules out any reaction at $\mathrm{C}_{5}$, but still allows the loss of water molecules from the $\mathrm{OH}$-terminated oligomers through a monomer transfer reaction.

\section{Influence of Solvents}

EG was extended with FO in different solvents at $1: 1$ $\mathrm{v} / \mathrm{v}$. The kinetics of FO consumption were followed by GC using the solvent as internal standard and gave much lower rates than the bulk reactions. As shown in Figure 11 , nitromethane gave the highest rate of conversion. The dielectric constant of the solvents used in these runs did not affect the kinetic behavior, as demonstrated by the fact that $\mathrm{CCl}_{4}(\varepsilon=2)$ and $\mathrm{CH}_{2} \mathrm{Cl}_{2}(\varepsilon=10)$ gave the same rates. The positive effect of nitromethane could

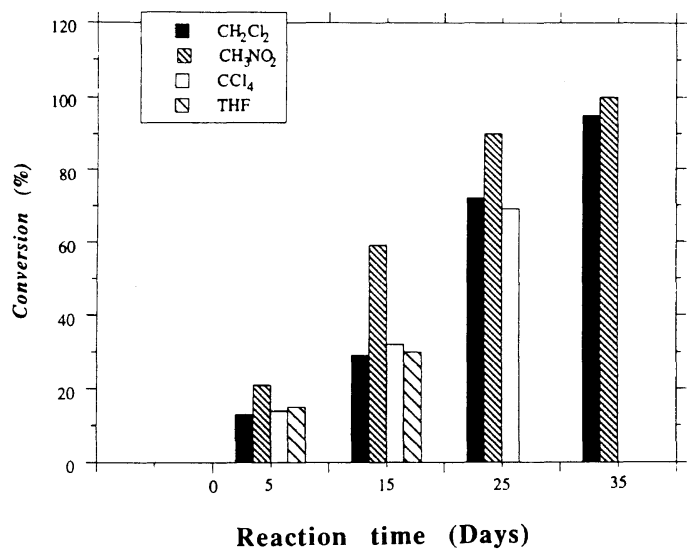

Figure 11. The rate of FO conversion in its reaction with $E G$ as a function of the medium: $\mathrm{FO} /$ solvent $=60 / 40(\mathrm{v} / \mathrm{v}) ;[\mathrm{FO}] /[\mathrm{EG}]=20$; $T=40^{\circ} \mathrm{C}$. The consumption of FO was monitored by GLC.

Table IV. Characteristics of THMP chain-extended by FMO and EG chain-extended by MFO.

\begin{tabular}{|c|c|c|c|c|c|c|}
\hline \multirow{2}{*}{ Oxirane } & \multirow{2}{*}{ Precursor } & \multirow{2}{*}{$\begin{array}{l}\text { Epoxide/ } \\
\text { precursor }\end{array}$} & Reaction time & \multirow{2}{*}{$M_{n}(\mathrm{th})^{\mathrm{a}}$} & \multirow{2}{*}{$M_{n}(\operatorname{det})^{\mathrm{b}}$} & \multirow{2}{*}{$F^{\mathrm{c}}$} \\
\hline & & & hours & & & \\
\hline \multirow[t]{4}{*}{ FMO } & THMP & 6 & 12 & 878 & 330 & 1.60 \\
\hline & & 12 & 12 & 1622 & 400 & 1.28 \\
\hline & & 20 & 24 & 2614 & - & - \\
\hline & & 40 & 48 & 5094 & - & - \\
\hline MFO & EG & 20 & 48 & 2614 & 300 & 1.95 \\
\hline
\end{tabular}

${ }^{a}$ Calculated from eq $1 .{ }^{b}$ Determined by VPO. ${ }^{c}$ Average $\mathrm{OH}-$ functionality calculated from eq 2. 
be attributed either to its much higher polarity $(\varepsilon=36)$ or to its acidic character. In the latter case a specific interaction could occur with the $\mathrm{OH}$ groups leading to an enhancement of their reactivity.

The characterization of these products by SEC, FTIR, and ${ }^{1} \mathrm{H}$ NMR after removal of the solvent revealed that working with solutions did not alter the structural features and the average chain length of the products.

The use of solvents with different donor-acceptor (DA) properties confirmed, as previously demonstrated, ${ }^{8}$ that the processes under scrutiny in this investigation are neither cationic nor anionic since they would not have occurred in tetrahydrofuran (THF) or $\mathrm{CCl}_{4}$, respectively. We reach therefore the same conclusion as in our study on the role of water in the polymerization of $\mathrm{FO}^{8}{ }^{8}$ viz., that the interactions in these systems are of a molecular nature. In other words the $\mathrm{OH}$ groups activate the oxirane ring of FO without any external catalysis and without the formation of ionic intermediates. The structure of the transition state is a matter of debate and represents a current topic in our laboratory.

\section{Influence of Temperature}

Carrying out the chain extention of EG by FO with $[\mathrm{FO}] /[\mathrm{EG}]=20$ at different temperatures showed that at $40^{\circ} \mathrm{C}$ the total conversion of $\mathrm{FO}$ was reached after 3 days, whereas at $70^{\circ} \mathrm{C}$, only $12 \mathrm{~h}$ were needed for this purpose. At $-5^{\circ} \mathrm{C}$ only $17 \%$ of $\mathrm{FO}$ were converted after 4 months. This gives an overall activation energy of about $75 \mathrm{~kJ} \mathrm{~mol}^{-1}$.

\section{CONCLUSION}

This study has led to two main conclusions, namely that the polymerization of 2-furyloxirane can be initiated by OH-bearing compounds, thus allowing chain extension, grafting and other functionalizations, but that transfer reactions seem unavoidable and this restricts the average length of the block thus introduced on substrates to 4 to 8 units. Therefore, the unique reactivity of FO and its corresponding potential as specific molecular reagent is confirmed, but the type of materials available by the very simple technique of mixing the hydroxy derivative with FO, without any added catalyst, is limited to structures containing relatively short "hairs" of PFO. The mechanism of the transfer reaction is also unique, compared with the behavior of classical systems, e.g., anionic polymerizations, involving FO and other oxiranes.

Acknowledgements. We wish to thank the FURCHIM Co. and the Agence Fraçaise pour la Maitrise de l'Energie for their financial support and the Laboratoire de Chimie des Agroressources of Toulouse National Polytechnic Institute for a gift of 2-furyloxirane.

\section{REFERENCES}

1. A. Gandini, in "Comprehensive Polymer Science," Suppl. vol., S. L. Aggarawal and S. Russo, Ed., Pergamon Press, Oxford, 1992, p 527.

2. A. Gandini and M. N. Belgacem, Prog. Polym. Sci., 22 (6), 1997.

3. A. Gandini, in "Encyclopedia of Polymer Science and Engineering," vol. 7, H. F. Mark, M. Bikales, C. G. Overberger, G. Menges, Ed., Wiley, New York, N.Y., 1988, p 454.

4. S. Boufi, M. N. Belgacem, J. Quillerou, and A. Gandini, Macromolecules, 26, 6706 (1993).

5. S. Boufi, A. Gandini, and M. N. Belgacem, Polymer, 36, 1689 (1995).

6. M. C. Salon, H. Amri, and A. Gandini, Polym. Commun., 31, 210 (1990).

7. H. Amri, M. N. Belgacem, and A. Gandini, Polymer, 37, 4815 (1996).

8. H. Amri, M. N. Belgacem, C. Signoret, and A. Gandini, Polym. Intern., 41, 427 (1996).

9. E. Borredon, M. Delmas, and A. Gaset, Tetrahedron, 17, 3945 (1988).

10. G. C. Levy and G. L. Nelson, "Carbon 13-Nuclear Magnetic Resonance in Organic Chemistry," 3rd ed, Wiley, New York, N.Y., 1972, p 38. 